Polish Journal of Microbiology

2012, Vol. 61, No 4, 311-313

SHORT COMMUNICATION

\title{
Nosocomial Outbreak due to Klebsiella pneumoniae at a Neurosurgery Unit
}

\author{
ALICJA SĘKOWSKA*, DOROTA KAMIŃSKA, BEATA NAKONOWSKA \\ and EUGENIA GOSPODAREK
}

Department of Microbiology, Nicolas Copernicus University, Collegium Medicum in Bydgoszcz, Poland

Received 11 October 2012, revised 1 September 2012, accepted 2 September 2012

\section{Abstract}

Thirty three isolates of K. pneumoniae were studied. The strains were cultured from different clinical specimens received from patients hospitalised at a Neurosurgery Unit at the Dr Jurasz University Hospital in Bydgoszcz. Production of ESBL was assessed using double disk synergy test. The genomic DNA was extracted from the strains separated by PFGE after digesting with XbaI endonuclease. Production of ESBL was detected in $81.8 \%$ of K. pneumoniae isolates. Molecular typing results revealed a great genetic diversity among K. pneumoniae isolates. All repeated PFGE patterns were detected in 12 (36.3\%) K. pneumoniae isolates.

Ke y words: Klebsiella pneumoniae, extended-spectrum beta-lactamases, nosocomial outbreak, pulsed-field gel electrophoresis

Klebsiella rods are Gram-negative bacteria which usually cause infections of respiratory tracts, urinary system or systemic infections. As opportunistic microorganisms, they mainly cause infections in patients with lower immunity and hospitalised at intensive care units, surgery units and paediatric units (Podschun and Ullmann, 1998). Klebsiella rods are the most common pathogen isolated from neurosurgical patients with meningitis (Tang etal., 1997). Gram-negative rods may produce different types of beta-lactamases. Strains which produce extended-spectrum betalactamases (ESBL) are a serious epidemiologic and therapeutic problem. To be more specific, therapeutic options against infections with ESBL-producing bacteria are limited because they are often coresistant to multiple non-beta-lactam antibiotics. K. pneumoniae rods, including ESBL-producers, may cause nosocomial outbreaks (Hernández et al., 2005; Peňa et al., 2001). The commonly applied phenotypic methods (biotyping, phagetyping, antimicrobial patterns) do not always provide a proper interpretation of an epidemiological situation at a hospital unit. Molecular methods allow for a genetic differentiation of strains belonging to the same species.

The purpose of this study was to evaluate genetic relatedness of K.pneumoniae strains isolated from patients hospitalised at a Neurosurgery Unit at the Dr Jurasz University Hospital in Bydgoszcz.
Thirty three clinical strains of K. pneumoniae were included. The strains were isolated from different specimens: wound swabs -12 , urine -11 , cerebrospinal fluid -8 and sputum -2 . Every strain came from other patient. The patients were hospitalised because of: aneurysm - $15(45.5 \%)$, brain tumour - $8(24.3 \%)$, meningitis $-4(12.2 \%)$, neuroinfections $-4(12.1 \%)$ and spinal fractures - (6.0\%). Eighteen (54.5\%) strains were cultured from men and 15 (45.5\%) from women, aged between 22 and 81 years. Twenty eight patients were treated with antibiotics. The strains were identified on the basis the results of biochemical reactions achieved in ID32E tests (bioMérieux). Production of ESBL was assessed using double disk synergy test (Gniadkowski et al., 2009). K. pneumoniae ATCC 700603 were used as reference strain. Macrorestriction analysis of chromosomal DNA with XbaI endonuclease (Fermentas) was carried out by pulsed-field gel electrophoresis (PFGE). PFGE was performed by using a CHIEF-MAPPER ${ }^{\mathrm{TM}}$ apparatus (Bio-Rad) with pulses ranging from 2 to $35 \mathrm{~s}$, at a voltage of $6 \mathrm{~V} / \mathrm{cm}$, at $14^{\circ} \mathrm{C}$ for $20 \mathrm{~h}$. As reference chromosomal DNA pattern Lambda Ladder standard (Bio-Rad) was used. The interpretation of PFGE patterns was according to the method of Tenover et al. (1995).

Out of all K. pneumoniae strains five biochemical profiles were detected. Two biotypes: 44074757331 (17 strains - 51.5\%) and 45074757331 (13 strains - 39.4\%) were prevailing. These two biotypes differentiated from

\footnotetext{
* Corresponding author: A. Sękowska, Department of Microbiology, Nicolas Copernicus University, Collegium Medicum in Bydgoszcz,
} Poland; phone: (48) 52 5853501; e-mail: ala.sek@wp.pl 




Fig. 1. PFGE fingerprinting of $\mathrm{XbaI}$ - digested DNA from K. pneumoniae strains $(\mathrm{n}=15)$

M - Lambda Ladder standard; 1-15 strain numbers

each other only by one biochemical reaction - the ability to urea hydrolysis. Out of all analysed K. pneumoniae strains, 27 (81.8\%) produced ESBLs. All K. pneumoniae strains isolated from cerebrospinal fluid were ESBL-positive. Ten K.pneumoniae strains cultured from wound swabs, eight strains from urine and 1 from sputum were also ESBL-positive.

Considering the results of PFGE testing, the studed strains were divided into four groups: identical -12 (36.5\% - group A), closely related $-2(6.0 \%$ - group B), potentially related - 1 (3.0\% - group C) (Fig. 1). Among K. pneumoniae strains, eighteen $(54.5 \%$ - group D) unrelated were detected (Fig. 2). Among K. pneumoniae strains belonging to the same genetic group, ESBLpositive and ESBL-negative phenotypes were detected. All genetically identical K. pneumoniae strains had the same biotype (44074757331). K.pneumoniae strains group A were isolated for a period of seven months from 5 men and 7 women. Ten isolates that belonged to clone A carried ESBL.

Of the Klebsiella genus, K. pneumoniae is the most common one to cause human infections. Klebsiella is usually found in the gastrointestinal tract in humans and animals. As it is known, colonisation can last very long and lead to infection. This mainly concerns hospital patients. K. pneumoniae are one of the most frequent ESBL producers, including Poland (Empel et al., 2008). The percentage of the K.pneumoniae ESBL-positive strains is varied and ranges from $7.5 \%$ to $44 \%$ (Falagas and Karageorgopoulos, 2009). In this study almost $82 \%$ of the K. pneumoniae ESBL-producing strains achieved. Such a high percentage might result from a specific character of the hospital unit, where patients within this study were hospitalised, from applied antibiotic prophylaxis and treatment in this institution as well as hygienic-sanitary activities. It is known that the use of the wide range cephalosporins affects the selection

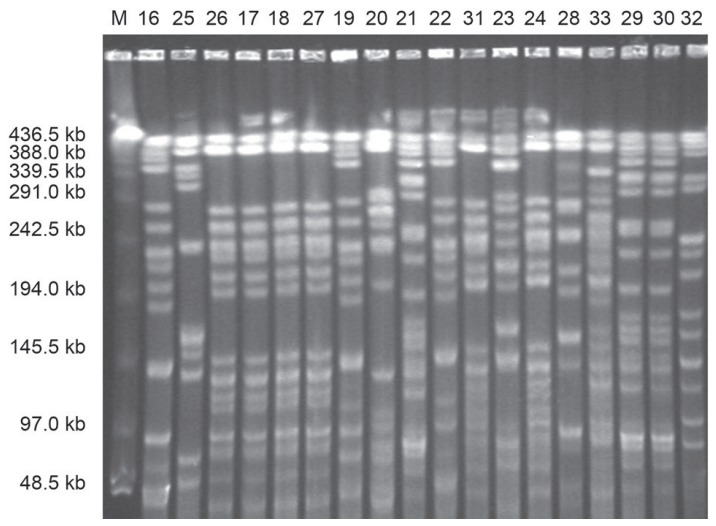

Fig. 2. PFGE fingerprinting of $\mathrm{XbaI}$ - digested DNA from K. pneumoniae strains $(\mathrm{n}=18)$

M - Lambda Ladder standard; 16-33 strain numbers

of the ESBL-positive strains (Morosini et al., 2006). In our study 19 patients were treated with third-generation cephalosporins. The selective pressure exerted by cephalosporins has been reported as a significant risk factor for the emergence of ESBL-producing K. penumoniae strains earlier (Peňa et al., 1997).

In this study K. pneumoniae strains group A were isolated for a period of 7 months with a one-month break from 12 patients. These strains had the same biotype, but differed with ESBL phenotype. On this ground it can be assumed that certain K. pneumoniae strains survive in the unit environment, in which stayed colonised patients or patients infected with these rods. Patient-to-patient transmission of ESBL-producing K. pneumoniae by hands of health care workers in the neurosurgery unit seemed to be possible. In the present study, we have used antibiotic sensitivity profiles and PFGE profiles as the typing methods. Antibiogram typing is one of the simplest and widely used method of bacteriological typing, although not comparable to genetic methods in discriminatory power. Molecular methods allow for a genetic differentiation of strains belonging to the same species.

In our study, PFGE showed the circulation of some different groups of K. pneumoniae at a Neurosurgery Unit. The analysed genetically identical K. pneumoniae strains have the same biochemical profiles, but different ESBL phenotype, so molecular typing should be supported with conventional diagnostics.

\section{Literature}

Empel J., A. Baraniak, E. Literacka, A. Mrówka, J. Fiett, E. Sadowy, W. Hryniewicz, M. Gniadkowski and the Beta-PL Study Group. 2008. Molecular survey of $\beta$-lactamases conferring resistance to newer $\beta$-lactams in Enterobacteriaceae isolates from Polish hospitals. Antimicrob. Agents Chemother. 52: 2449-2454. 
Falagas M.E. and D.E. Karageorgopoulos. 2009. Extended-spec trum $\beta$-lactamase producing organisms. J. Hosp. Inf. 73: 345-354. Gniadkowski M., D. Żabicka and W. Hryniewicz. 2009. Recomended testes for antibiotics and chemotheraputics bacterial sen sitivity. Estimation Gram-negative rod shape bacteria sensitivity (in Polish). www.korld.edu.pl/pdf/02-Rek2009-Paleczki z rodziny Enterobacteriaceae.pdf

Hernández J.R., L. Martínez-Martínez, R. Cantón, T.M. Coque A. Pascual and the Spanish Group for Nosocomial Infection (GEIH). 2005. Nationwide study of Escherichia coli and Klebsiella pneumoniae producing organism at a tertiary care hospital. J Antimicrob. Chemother. 49: 2122-2125.

Morosini M.I., M. Garcia-Castilio, T.M. Coque, V. Aránzazu, Ă. Novais, E. Loza, F. Baquero and R. Cantón. 2006. Antibiotic coresistance in extended-spectrum $\beta$-lactamase producing Enterobacteriaceae and in vitro activity of tigecycline. Antimicrob. Agents Chemother. 50: 2695-2699.
Peňa C., M. Pujol, C. Ardanuy, A. Ricart, R. Pallares, J. Linares, J. Ariza and F. Gudiol. 2001. An outbreak of hospital-acquired Klebsiella pneumoniae bacteriemia, including strains producing extended-spectrum beta-lactamase. J Hosp. Infect. 47: 53-59.

Peňa C., M. Pujol, A. Ricart, C. Ardanuy, J. Ayats, J. Linares, F. Garrigosa, J. Ariza and F. Gudiol. 1997. Risk factor for faecal carriage of Klebsiella pneumoniae producing extended-spectrum beta-lactamase in the intensive care unit. J. Hosp. Infect. 35: 9-16 Podschun R. and U. Ullmann. 1998. Klebsiella spp. as nosocomial pathogens: epidemiology, taxonomy, typing methods and pathogenicity factors. Clin. Microbiol. Rev. 11: 589-603.

Tang L.M., S.T. Chen, W.C. Hsu and C.M. Chen. 1997. Klebsiella meningitis in Taiwan: an overview. Epidemiol. Infect. 119: 135-142. Tenover F.C., R.D. Arbeit, R. Goering, P.A. Mickelsen, D.H. Persing and B. Swaminathan. 1995. Interpreting chromosomal DNA restriction patterns produced by pulsed-field gel electrophoresis: criteria for bacterial strain typing. J. Clin. Microbiol. 33: 2233-2239. 